\title{
Papers
}

\section{Progression to symptomatic disease in people infected with HIV-1 in rural Uganda: prospective cohort study}

Dilys Morgan, Cedric Mahe, Billy Mayanja, James A G Whitworth

\begin{abstract}
Objectives To estimate the rate of progression from seroconversion to symptomatic disease in adults infected with HIV-1, and to establish whether the background level of signs and symptoms commonly associated with HIV-1 in uninfected controls are likely to affect progression rates.

Design Longitudinal, prospective cohort study of people infected with HIV-1 and randomly selected subjects negative for HIV-1 antibodies identified during population studies.

Setting Study clinic with basic medical care in rural Uganda.

Subjects 275 patients infected with HIV-1 (107

prevalent cases and 168 incident cases) and 246 controls negative for HIV-1 antibodies.

Main outcome measures Signs and symptoms of HIV disease, as determined by stages 2 and 3 of the World Health Organization clinical staging system. Results The median time from seroconversion to WHO stage 2 was 25.4 months and to stage 3 was 45.5 months. $43 \%$ of the participants infected with HIV-1 had signs or symptoms by two years after seroconversion. The most common clinical conditions used to define progression of disease were weight loss, mucocutaneous manifestations, bacterial infections, chronic fever, and chronic diarrhoea. Although the rates of these conditions (apart from minor weight loss) were significantly higher in the participants infected with HIV-1, they were also relatively frequent in the control group. Herpes zoster, oral candidiasis, and pulmonary tuberculosis were not common events in the control group and therefore were more indicative of infection with HIV-1.

Conclusions Disease progression associated with infection with HIV-1 seems to be rapid in rural Uganda. This is most likely due to the high prevalence of conditions in the general population that could be taken as symptoms and signs of infection with HIV-1.
\end{abstract}

\section{Introduction}

Although some studies have found that progression of disease in patients infected with HIV-1 is more rapid in African people living in sub-Saharan Africa, ${ }^{1-4}$ most studies have found rates of progression similar to those in rich countries. ${ }^{5-9}$ However, in Africa it is rare to find cohorts for whom tests negative for HIV-1 antibody and positive HIV-1 tests (and hence an estimated date of seroconversion) are documented.

We report rates of, and reasons for progression to, early symptomatic disease in adults for whom estimated dates of HIV-1 seroconversion were available in a cohort in rural Uganda. We also assessed the rates of conditions commonly associated with HIV-1 infection in participants infected with HIV-1 before they developed AIDS compared with those in participants not infected with HIV-1.

\section{Methods \\ Participants and design}

We established a clinical cohort in rural south western Uganda in 1990 by recruiting participants from a large study that followed the dynamics of HIV-1 infection with annual HIV-1 serosurveys of the general population. ${ }^{10}$ The cohort comprised prevalent cases of HIV-1 infection diagnosed in 1989 and 1990 and incident cases detected during annual serosurveys; we used randomly selected people who were negative for HIV-1 antibodies, as controls. ${ }^{11}$ The estimated date of seroconversion for the incident cases was taken as the midpoint between the last test negative result and first positive result for antibodies to HIV-1.

All participants gave informed written consent (signature or thumbprint), and they were reviewed by a study doctor every three months. The participants were asked about their medical history, were given a full physical examination, and underwent basic laboratory investigations. Any complaints or medical findings were investigated and treated with drugs from the World Health Organization's list of essential drugs. Participants could also attend the clinic at any time when they were ill during the study. At each routine visit, participants infected with HIV-1 were categorised according to the clinical and performance scale of the staging system for patients infected with HIV-1 proposed by the WHO (see box). ${ }^{12}$

The staff at the clinic were not aware of the HIV status of the participants. This ensured confidentiality and reduced the risk of bias in the reports. All participants were strongly encouraged to attend the HIV counselling sessions and testing facilities that were provided as part of the study.

\author{
Medical Research \\ Council \\ Programme on \\ AIDS, Uganda \\ Virus Research \\ Institute, \\ PO Box 49, \\ Entebbe, Uganda \\ Dilys Morgan \\ clinical epidemiologist \\ Cedric Mahe \\ statistician \\ Billy Mayanja \\ medical officer \\ James A G \\ Whitworth \\ head of programme \\ Correspondence to: \\ D Morgan, Public \\ Health Laboratory \\ Service \\ Communicable \\ Disease \\ Surveillance Centre, \\ London NW9 5EQ \\ dmorgan@phls. \\ org.uk
}

BMJ 2002;324:193-7 


\section{Statistical analysis}

To minimise the number of missed events when the median time from seroconversion to symptomatic disease, represented by WHO stages 2 and 3, was estimated, we included only incident cases seen within two years of their estimated date of seroconversion.

\section{Proposed WHO staging system for patients infected with HIV-1 ${ }^{12}$}

Stage 1:

- Asymptomatic

- Persistent generalised lymphadenopathy

Stage 2:

- Weight loss between $5 \%$ and $10 \%$ of body weight

- Minor mucocutaneous manifestations (seborrhoeic dermatitis, prurigo, fungal nail infections, recurrent oral ulcerations, angular stomatitis)

- Herpes zoster within the past five years

- Recurrent upper respiratory tract infections (for example, bacterial sinusitis)

And/or

- Performance scale 2: symptomatic, normal activity

Stage 3:

- Weight loss $>10 \%$ body weight

- Unexplained chronic diarrhoea for longer than one month

- Unexplained prolonged fever (intermittent or constant) for longer than one month

- Oral candidiasis

- Oral hairy leukoplakia

- Pulmonary tuberculosis within last year

- Severe bacterial infections (for example, pneumonia, pyomyositis)

And/or

- Performance scale 3: bedridden for less than $50 \%$ of the day during the last month

Clinical stage 4 (AIDS):

- HIV wasting syndrome*

- Pneumocystis carinii pneumonia

- Toxoplasmosis of the brain

- Cryptosporidiosis with diarrhoea for more than one month

- Cryptococcus, extrapulmonary

- Cytomegalovirus infection of an organ other than liver, spleen, or lymph nodes

- Herpes simplex virus infection-mucocutaneous for more than 1 month or visceral of any duration

- Progressive multifocal leukoencephalopathy

- Any disseminated endemic mycosis

- Candidiasis of the oesophagus, trachea, bronchi, or lungs

- Atypical mycobacteriosis, disseminated

- Non-typhoidal salmonella septicaemia

- Extrapulmonary tuberculosis

- Lymphoma

- Kaposi's sarcoma

- HIV encephalopathy†

And/or

- Performance scale 4 : bedridden for more than $50 \%$ of the day during last month

*Defined by the Centers for Disease Control and Prevention as weight loss of greater than 10\% body weight, plus either unexplained chronic diarrhoea (greater than 1 month) or chronic weakness and unexplained prolonged fever (greater than 1 month). $\dagger$ Defined by the Centers for Disease Control and Prevention as clinical findings of disabling cognitive dysfunction and/or motor dysfunction, interfering with activities of daily living, progressing over weeks to months in the absence of a concurrent illness or condition other than infection with HIV that could explain the findings.

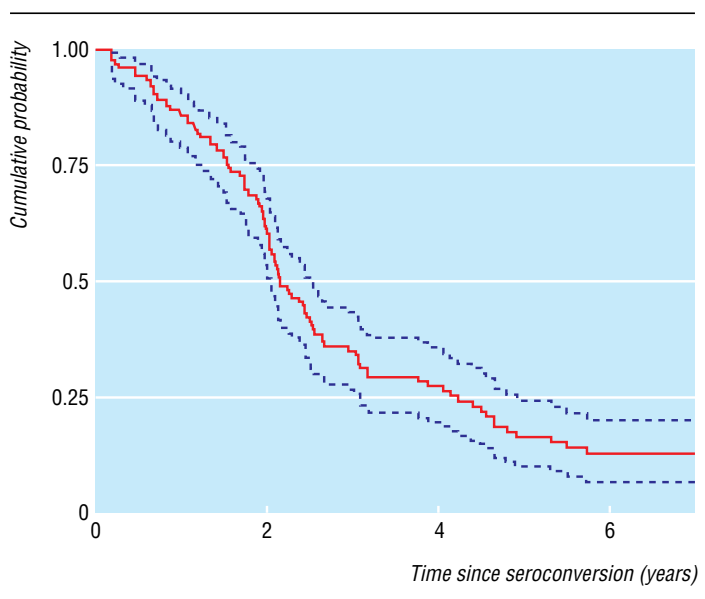

Cumulative probability (95\% confidence intervals) of remaining symptom-free from time of seroconversion (Kaplan-Meier plot)

The WHO staging system is hierarchical-it is assumed that once individuals have moved to a given stage, they have passed though all previous stages. Participants who had symptoms at enrolment or who progressed directly to a higher stage were considered to be left censored (the event that defined the move to the next stage happened before the date of recording). This left censoring tends to overestimates of the time from seroconversion to a given stage. We provide the proportion of individuals who were left censored for each stage so that the degree of bias can be assessed. The date of the routine appointment at which a participant was first seen with an event that defined a WHO stage was used to estimate the time between seroconversion and the patient developing symptoms.

Kaplan-Meier survival methods were used to estimate the median probabilities (and interquartile ranges) and cumulative probabilities (95\% confidence intervals by Greenwood's method) for times to the various endpoints. To estimate the background level of stage defining conditions in this population, we compared the rates of conditions during follow up-from the first visit to the last visit before the patient was classified as having AIDS-for all incident cases, prevalent cases and controls by using the MantelHaenszel test. All analyses were performed with the STATA 6.0 statistical package. This paper reports data from 1990 to the end of 2000 .

\section{Results}

By the end of 2000, 275 patients infected with HIV-1 (107 prevalent cases and 168 incident cases) and 246 controls who tested negative for HIV-1 infection had been enrolled. We enrolled more than $80 \%$ of individuals identified for selection from the large population study into the clinical cohort, and over $92 \%$ of participants living in the area were seen during 2000. Compliance rates remained high.

In total, 142 incident cases were seen within two years of their estimated date of seroconversion (table 1). Figure 1 shows the cumulative probabilities of remaining symptom free after seroconversion.

The most common clinical conditions associated with disease progression were weight loss, mucocutaneous manifestations, bacterial infections, chronic 
fever, and chronic diarrhoea (table 1). The number of events and the rates of these and other conditions defining a WHO stage (prior to AIDS) in all participants are shown in table 2 . With the exception of minor weight loss, the rates of these conditions were significantly higher in participants infected with HIV-1 than in controls, although the conditions were also relatively frequent in the control group. Herpes zoster, oral candidiasis, and pulmonary tuberculosis were not common events in the control participants, as shown by their higher rate ratios.

\section{Discussion}

Early manifestations and progression rates of disease in patients infected with HIV-1 are particularly difficult to assess in Africa. Most reports of clinical manifestations have been in patients admitted to hospital or attending clinics; these patients are not representative of the general population, because patients tend to present late and few make it to referral centres.

\section{Time between seroconversion and appearance of} symptoms

Symptoms of infection with HIV-1 seem to developed quickly in incident cases seen within two years of their estimated date of seroconversion: only $17 \%$ of participants remained symptom-free five years after seroconversion. The median time to WHO stage 2 (25.4 months) was probably overestimated due to the effects of left censoring, but the bias for time to WHO stage 3 (45.5 months) should be negligible. Other studies in Kenya and Haiti have also reported rapid progression from seroconversion to symptomatic disease. ${ }^{13}{ }^{14}$ Progression times in industrialised countries are much longer, with $78 \%-85 \%$ of patients infected with HIV remaining asymptomatic five years after seroconversion. $^{15} 16$

Recognising progression to symptomatic disease

The most common single reason for recognising progression to symptomatic disease in our cohort was minor weight loss, but this was as common in controls who tested negative for HIV-1 antibodies as in participants infected with HIV-1. Minor mucocutaneous
Table 1 Enrolment characteristics of the incident cases infected with HIV-1, used for estimating progression times, median time from seroconversion to WHO stages 2 and 3 and reasons for progression

\begin{tabular}{|c|c|}
\hline Characteristic & Value \\
\hline No of participants seen within two years of seroconversion & 142 \\
\hline Men & 70 \\
\hline Women & 72 \\
\hline Median (IQR) age (years) & $30(24-38)$ \\
\hline Median (IQR) time between tests negative and positive for HIV antibodies (months) & $12.1(9.8-18.0)$ \\
\hline Median (IQR) time from seroconversion to enrolment (months) & $10.6(7.3-16.8)$ \\
\hline Median (IQR) follow up from seroconversion to last visit (years) & $4.2(2.3-6.6)$ \\
\hline \multicolumn{2}{|l|}{ No of patients in each WHO stage on enrolment: } \\
\hline 1 (asymptomatic) & 123 \\
\hline 2 (mild disease) & 12 \\
\hline 3 (moderate disease) & 6 \\
\hline 4 (AIDS) & 1 \\
\hline Median $(95 \% \mathrm{Cl})$ time from seroconversion to stage 2 (months) ${ }^{*}$ & $25.4(23.9$ to 29.6$)$ \\
\hline No (\%) of patients in WHO stage 2 & 63 \\
\hline \multicolumn{2}{|l|}{ Reasons for progression: ${ }^{* \star}$} \\
\hline Minor weight loss (5-10\% baseline weight) & $38(61)$ \\
\hline Minor mucocutaneous manifestations & $16(25)$ \\
\hline Herpes zoster & $9(14)$ \\
\hline Median $(95 \% \mathrm{Cl})$ time from seroconversion to stage 3 (months) $\dagger$ & $45.5(36.2$ to 51.3$)$ \\
\hline No (\%) of patients in WHO stage 3 & 72 \\
\hline \multicolumn{2}{|l|}{ Reasons for progression: ${ }^{* \star}$} \\
\hline Major weight loss $(>10 \%)$ & $24(33)$ \\
\hline Severe bacterial infection & $23(32)$ \\
\hline Chronic diarrhoea & $13(18)$ \\
\hline Chronic fever & $10(14)$ \\
\hline Pulmonary tuberculosis & $3(4)$ \\
\hline Oral candidiasis & $2(3)$ \\
\hline Oral hairy leukoplakia & $1(1)$ \\
\hline
\end{tabular}

IQR=interquartile range.

*Using 104 subjects in stage 2: 51 progressed from stage 1; 12 were in stage 2 and 7 were in higher stages on enrolment; and 34 went straight to stages 3 or 4 . Thus $53(51 \%)$ were left censored.

**Each participant could have more than one reason for progression.

†Using 80 subjects in stage 3: 66 progressed from stages 1 or 2; 6 in stage 3 and 1 in stage 4 on enrolment; and 7 went straight to stage 4 . Thus $14(18 \%)$ were left censored.

problems, severe bacterial infections, chronic fever, and chronic diarrhoea were also common clinical complaints that occurred before a patient was classified as having AIDS. Although participants infected with HIV-1 had significantly higher rates of these conditions, they were also frequent in the controls (see table 2). However, in agreement with other African studies, ${ }^{17} 18$ we found that herpes zoster, oral candidia-

Table 2 Rates of visits to study clinic by patients with conditions defining WHO stages (pre-AIDS) in participants infected with HIV-1 compared with controls negative for HIV-1 antibodies

\begin{tabular}{|c|c|c|c|c|c|c|}
\hline & \multicolumn{2}{|c|}{$\begin{array}{l}\text { Infected with HIV } \\
\text { (total PYO=1120.2) }\end{array}$} & \multicolumn{2}{|c|}{$\begin{array}{l}\text { Negative for HIV antibodies } \\
\text { (total PYO=1336.5) }\end{array}$} & \multirow[b]{2}{*}{ Rate ratio* $(95 \% \mathrm{CI})$} & \multirow[b]{2}{*}{$P$ value } \\
\hline & $\begin{array}{c}\text { No of } \\
\text { patients }\end{array}$ & $\begin{array}{c}\text { Rate (per } 100 \\
\text { PYO) }\end{array}$ & $\begin{array}{c}\text { No of } \\
\text { patients }\end{array}$ & $\begin{array}{l}\text { Rate (per } \\
100 \text { PYO) }\end{array}$ & & \\
\hline \multicolumn{7}{|l|}{ WHO stage 2: } \\
\hline Weight loss $(5 \%-10 \%)$ & 521 & 46.5 & 551 & 41.2 & $1.1(1.0$ to 1.3$)$ & 0.045 \\
\hline Minor mucocutaneous disease & 197 & 17.6 & 58 & 4.3 & $4.1(3.0$ to 5.4$)$ & $<0.001$ \\
\hline Herpes zoster & 51 & 4.6 & 6 & 0.5 & 10.1 (4.4 to 28.9$)$ & $<0.001$ \\
\hline Recurrent upper respiratory tract infection & 21 & 1.9 & 7 & 0.5 & $3.6(1.5$ to 8.4$)$ & 0.002 \\
\hline \multicolumn{7}{|l|}{ WHO stage 3: } \\
\hline Weight loss $>10 \%$ & 232 & 20.7 & 98 & 7.3 & $2.8(2.2$ to 3.6$)$ & $<0.001$ \\
\hline Chronic diarrhoea & 60 & 5.4 & 22 & 1.6 & $3.3(2.0$ to 5.3$)$ & $<0.001$ \\
\hline Prolonged fever & 56 & 5.0 & 37 & 2.8 & $1.8(1.2$ to 2.7$)$ & 0.005 \\
\hline Oral candidiasis & 43 & 3.8 & 1 & 0.08 & 51.3 (7.1 to 372$)$ & $<0.001$ \\
\hline Oral hairy leukoplakia & 6 & 0.5 & 1 & 0.08 & 7.2 (0.9 to 59.5$)$ & 0.033 \\
\hline Pulmonary tuberculosis & 24 & 2.1 & 2 & 0.08 & 28.6 (3.9 to 212$)$ & $<0.001$ \\
\hline Severe bacterial infection & 126 & 11.3 & 47 & 3.5 & $3.2(2.3$ to 4.5$)$ & $<0.001$ \\
\hline
\end{tabular}

PYO=person years of observation.

${ }^{*}$ Rate in patients infected with HIV-1 divided by rate in participants negative for HIV-1 antibodies.

†Comparison of rate ratios using Mantel-Haenszel test. 


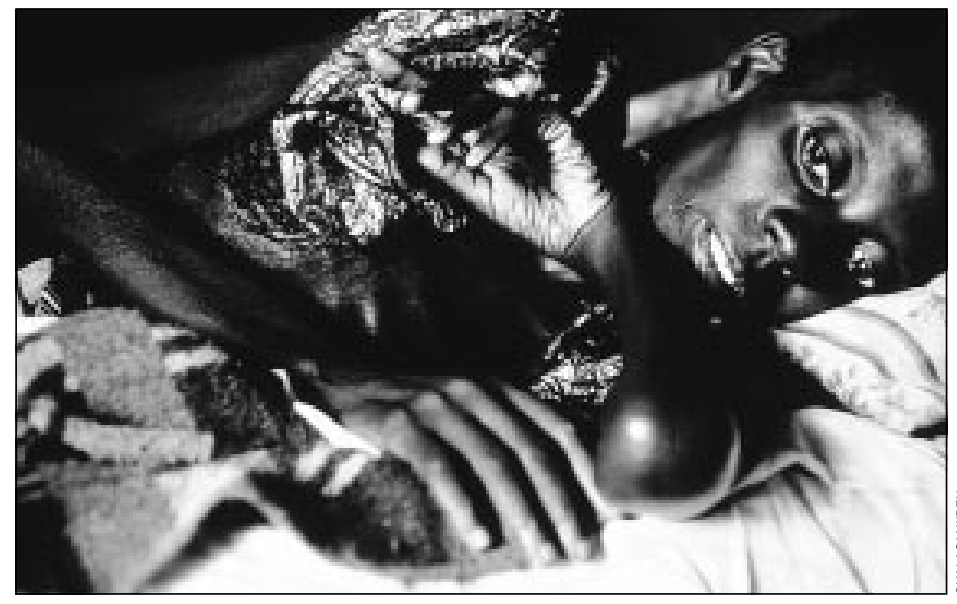

sis, and pulmonary tuberculosis were highly predictive for infection with HIV-1. The small number of participants may have affected our results, but a study of women in Rwanda reported similar rates of weight loss, mucocutaneous conditions, chronic fever, and diarrhoea in groups whose test results were positive and negative for HIV-1 antibodies. ${ }^{6}$ In rich countries, the rates of these conditions, and the rate ratios, were considerably lower in groups testing positive and negative for HIV-1 antibodies. ${ }^{19} 20$

\section{Background levels of other conditions}

Most of the population in rural Uganda lives in poverty; food is often in limited supply, there is no electricity, and there is poor access to any, let alone clean, water. Malaria is endemic, and infections other than HIV, especially bacterial infections, are common. The shorter interval from seroconversion to symptomatic disease in African populations probably reflects the high background level of these conditions, rather than rapid disease progression.

\section{Conclusions}

The progression of disease in patients infected with HIV-1 in Africa seems to be rapid; this is most likely to be due to the high prevalence of what could be taken as symptoms and signs of infection with HIV-1 in the general population. Studies that showed rapid progression of disease in patients infected with HIV in Africa could have led to the belief that HIV disease progresses more rapidly in Africa than elsewhere.

We thank all the staff in the study clinic and in Entebbe who supported our work. Most importantly, we thank the participants.

Contributors: DM was the project leader and designed and conducted the study. CM maintained the databases and helped with the data analysis. BM was responsible for data collection and clinical care of the participants. JAGW assisted with the design and interpretation of the study. DM and JAGW are guarantors for the paper.

Funding: Medical Research Council and the Department for International Development.

Competing interests: None declared.

1 Colebunders R, Ryder R, Francis H, Nekwei W, Bahwe Y, Lebughe I, et al. Seroconversion rate, mortality and clinical manifestations associated with the receipt of a human immunodeficiency virus-infected blood the receipt of a human immunodeficiency virus-1
transfusion in Kinshasa, Zaire. J Infect Dis 1991;164:450-6.

2 N'Galy B, Ryder RW, Kapita B, Mwandagalirwa K, Colebunders RL, Francis $\mathrm{H}$, et al. Human immunodeficiency virus infection among employees in an African hospital. N Engl J Med 1988;319:1123-7.

3 Whittle H, Egboga A, Todd J, Corrah T, Wilkins A, Demba E, et al. Clinical and laboratory predictors of survival in Gambian patients with symptomatic HIV-1 or HIV-2 infection. AIDS 1992;6:685-9.

\section{What is already known on this topic}

The few studies that have reported time from seroconversion to HIV-1 symptomatic disease in poor countries suggest that this interval is shorter than in rich countries

\section{What this study adds}

Progression from seroconversion to symptomatic disease seems to be rapid in rural Africa

The apparent rapid disease progression in rural Africa is most likely to be due to the high prevalence of what could be taken as symptoms and signs of HIV-1 in the general population

4 Anzala OA, Nakelkerke NJD, Bwayo JJ, Holton D, Moses S, Ngugi EN, et al. Rapid progression to disease in African sex workers with human immunodeficiency virus type 1 infection. JInfect Dis 1995;171:686-9.

5 Mann JM, Bila K, Colebunders RL, Kalemba K, Khonde N, Bosenge N, et al. Natural history of human immunodeficiency virus infection in Zaire. Lancet 1986;ii:707-9.

6 Leroy V, Msellati P, Lepage P, Batungwanayo J, Hitmana DG, Taelman H, et al. Four years of natural history of HIV-1 infection in African women: a prospective cohort study in Kigali (Rwanda), 1988-1993. J Acquir Immune Defic Syndr 1995:9:415-21.

7 Kerlikowske KM, Katz MH, Allen S, Wolf W, Hudes HS, Karita E, et al. $\beta_{2}$-microglobulin as a predictor of death in HIV-infected women from Kigali, Rwanda. AIDS 1994;8:963-9.

8 French N, Mujugira A, Nakiyingi J, Mulder D, Janoff EN, Gilks CF Immunological and clinical stages in HIV-1 infected adults are comparable and provide no evidence of rapid progression but poor survival with advanced disease. J Acquir Immune Def Syndr 1999;22:509-16.

9 Marlink R, Kanki P, Thior I, Travers K, Eisen G, Siby T, et al. Reduced rate of disease development after HIV-2 infection as compared to HIV-1. Science 1994;265:1587-90.

10 Kamali A, Carpenter LM, Whitworth JAG, Pool R, Ruberantwari A, Ojwiya A. Seven-year trends in HIV-1 infection rates, and changes in sexual behaviour among adults in rural Uganda. AIDS 2000;14:427-34.

11 Morgan D, Malamba S, Maude G, Okongo M, Wagner HU, Mulder D, et al. An HIV-1 natural history cohort and survival times in rural Uganda. AIDS 1997;11:633-40.

12 World Health Organization: acquired immune deficiency syndrome (AIDS): interim proposal for a WHO staging system for HIV-1 infection and disease. Whly Epidemiol Rec 1990;65:221-8.

13 Nagelkerke NJD, Plummer FA, Holton D, Anzala AO, Manji F, Ngugi EN, et al. Transition dynamics of HIV disease in a cohort of African prostitutes: a Markov model approach. AIDS 1990;4:743-7.

14 Deschamps M-M, Fitzgerald DW, Pape JW, Johnson WD. HIV infection in Haiti: natural history and disease progression. AIDS 2000;14:2515-21.

15 Flegg PJ. The natural history of HIV infection: a study in Edinburgh drug users. J Infect 1994;29:311-21

16 Lee CA, Phillips AN, Elford J, Janossy G, Griffiths P, Kernoff P. Progression of HIV disease in a haemophilic cohort followed for 11 years and the effect of treatment. BMJ 1991;303:1093-6.

17 Lindan CP, Allen S, Serufilira A, Lifson AR, Van de Perre P, Chen-Rundle A, et al. Predictors of mortality among HIV-infected women in Kigali, Rwanda Ann Intern Med 1992;116:320-8.

18 Miller WC, Thielman NM, Swai N, Cegielski JP, Shao J, Manyenga D, et al. Diagnosis and screening of HIV/AIDS using clinical criteria in Tanzanian adults. J Acquir Immune Def Syndr 1995;9:408-14.

19 Holmberg SD, Buchbinder SP, Conley LJ. The spectrum of medical conditions and symptoms before acquired immunodeficiency syndrome in homosexual and bisexual men infected with the human immunodeficiency virus. Am J Epid 1995;141:395-404.

20 Brettle RP, Foreman A, Povey S. Clinical features of early HIV in the Edinburgh city cohort. Intern J AIDS STD 1996;7:110-6. (Accepted 11 December 2001)

\section{Endpiece \\ The danger of health books}

Be careful of reading health books, you may die of a misprint.

Mark Twain

Submitted by Peter Teichman, assistant professor, Department of Family Medicine, West Virginia University 


\title{
Commentary: Virus, host, or environment?
}

\author{
Peter H Kilmarx
}

Morgan et al present data from a fruitful, long running body of research that looked at the clinical manifestations of infection with HIV-1 in a cohort in rural Uganda. Their key finding was that symptoms suggestive of infection with HIV-1 develop relatively rapidlythe median time from seroconversion to symptomatic disease (WHO stage 2) was only 25.4 months. In contrast, most patients in rich countries are asymptomatic even five years after acquiring infection.

Does disease associated with infection with HIV-1 progress more quickly in African populations? This is a crucial issue. Knowing the rate of disease progression allows us to give a prognosis to individual patients. At the population level, it helps us to understand the broader impact of the epidemic on population characteristics, the workforce, and healthcare needs. Rates of disease progression might differ between populations for three reasons: variations in the pathogenicity of the subtypes of HIV-1 that cause infections, variations in host characteristics, and the presence or absence of certain environmental factors-such as the presence of coexistent Mycobacterium tuberculosis or the availability of adequate medical treatment. These factors are difficult to disentangle, but in developed countries, rates of disease progression do not seem to be associated with the viral subtype or the patient's country of origin. ${ }^{1}$ Data from poor countries are sparse and conflicting, but some show that the progression of disease in these settings is more rapid than in rich parts of the world. ${ }^{23}$ More recently, the use of highly active antiretroviral drugs has become the overwhelming factor that has affected disease progression. In 2001 the estimated number of deaths from HIV and AIDS in North America, western Europe, Australia, and New Zealand was 75500 (out of a total population of about 731 million); this compares with 2.3 million deaths in sub-Saharan Africa (total population about 596 million). ${ }^{4}$

This report from Morgan et al indicates a key reason for the apparently rapid development of symptoms in people in rural Uganda: many of the study's participants who were negative for HIV-1 antibodies also had symptoms suggestive of infection with
HIV-1. The most commonly reported condition-loss of $5-10 \%$ of body weight-occurred at a rate of more than 40 per 100 person years of follow up whether or not participants were infected with HIV-1. Other symptoms, such as prolonged fever, were more common in the people infected with HIV-1, but they were also common among those who were negative for HIV-1 antibodies. The rapid development of symptoms among rural Ugandans infected with HIV-1 is, at least in part, unrelated to infection, but it is linked to the poor background health status of this population.

This study underscores two points. Firstly, the presence of symptoms is a poor guide for staging disease associated with HIV-1 infection. "Harder" outcomes, such as CD4 T lymphocyte count, blood concentration of HIV RNA, and death, are better endpoints on which to base epidemiological studies of disease progression. However, these laboratory tests are expensive and not available for patients in poor settings. Accurate, low cost assays to monitor disease activity and progression are needed urgently. ${ }^{5}$ Secondly, the health status of rural Africans is generally poor. Further research is needed to determine the role of the environment in the progression of disease associated with infection with HIV-1 in poor settings. Meanwhile, it is appropriate that initiatives to provide antiretroviral treatment, which are much needed, also aim to improve basic health care and control other infectious diseases, such as malaria and tuberculosis.

Competing interests: None declared.

1 Del Amo J, Petruckevitch A, Phillips A, Johnson AM, Stephenson J, Desmond N, et al. Disease progression and survival in HIV-1-infected Africans in London. AIDS 1998;12:1203-9.

2 Deschamps M-M, Fitzgerald DW, Pape JW, Johnson WD. HIV infection in Haiti: natural history and disease progression. AIDS 2000;14:2515-21.

3 Kilmarx PH, Limpakarnjanarat K, Kaewkungwal J, Srismith R, Saisorn S, Uthaivoravit W, et al. Disease progression and survival with human immunodeficiency virus type 1 subtype $\mathrm{E}$ infection among female sex workers in Thailand. J Infect Dis 2000;181:1598-606.

4 Joint United Nations Programme on HIV/AIDS (UNAIDS). AIDS epidemic update: December 2001. Geneva: World Health Organization, 2001.

5 Schupbach J, Boni J, Flepp M, Tomasik Z, Joller H, Opravil M. Antiretroviral treatment monitoring with an improved HIV-1 p24 antigen test: an inexpensive alternative to tests for viral RNA.J Med Virol 2001;65:225-32.
Thailand

MOPH-US CDC

Collaboration,

Ministry of Public

Health, Nonthabur 11000 , Thailand

Peter H Kilmarx associate director for science

pbk4@cdc.gov

\section{One hundred years ago \\ Is the lady doctor a failure?}

This question has recently been asked in America and in one important quarter at least has been answered in the affirmative. This is all the more remarkable, since America is not only the country which witnessed the new birth of the lady doctor in modern times, but that in which she has hitherto appeared most to flourish. Miss Elizabeth Blackwell, who was the first, graduated in 1849. Three years later there were six in Philadelphia. In 1889 there were 3,000 lady doctors in the United States; in 1896 there were 4,555 , and now there are probably 6,000 , some of whom have very lucrative practices. But these would seem to be the exception. From Chicago comes the news that Princess Bamha Dhuleep Singh, daughter of the late Maharajah Dhuleep Singh, and seventy other young women, who were studying medicine at the North-Western University, will have to seek another school. After thirty two years' trial the trustees of the University have come to the conclusion that women are not a success as doctors. The school is to be abolished and the property sold. One of the trustees, Mr. Raymond, goes so far as to say that it is impossible to make a doctor of a woman, He adds: "We have run the Women's Medical School at a loss of $£ 5,000$ a year. Women cannot grasp chemical laboratory work or the intricacies of surgery. Fifteen years ago the graduating class of men and women signed a memorial saying that coeducation was a failure. Then we conducted the college exclusively for women, and it has been worse than a failure."

(BMJ 1902;i:287) 